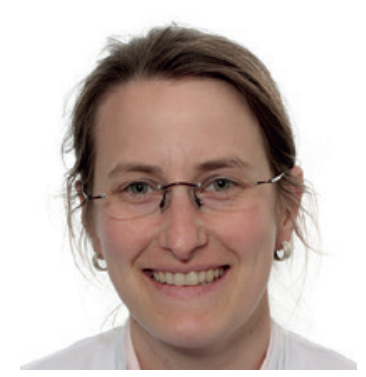

\title{
COVID-19: Wann muss ich an MIS-A denken?
}

\author{
Stefanie Keymel
}

Universitätsklinikum Düsseldorf, Klinik für Kardiologie, Pneumologie und Angiologie, Düsseldorf, Deutschland

Abstract aus Ahmad F, Ahmed A, Rajendraprasad S, et al.: Multisystem inflammatory syndrome in adults: A rare sequela of SARS-CoV-2 infection. International Journal of Infectious Diseases 108 (2021) 209-211.

\section{Keywords}

Multisystem inflammatory syndrome in adults (MIS-A) .

SARS-CoV-2 infection · COVID-19

\begin{abstract}
Multisystem inflammatory syndrome in adults (MIS-A) came to attention back in June 2020, when the United States Center for Disease Control and Prevention (CDC) received initial reports regarding patients who had presented delayed and multisystem involvement of the disease, with clinical course resembling multisystem inflammatory syndrome in children (MIS-C). This study introduces a case of MIS-A, where the patient presented 3 weeks
\end{abstract}

after initial COVID-19 exposure. His clinical course was consistent with the working definition of MIS-A as specified by the CDC. Aggressive supportive care in the intensive care unit, utilization of advanced heart failure devices, and immunomodulatory therapeutics (high-dose steroids, anakinra, intravenous immunoglobulin) led to clinical recovery. Management of MIS-A is a topic of ongoing research and needs more studies to elaborate on treatment modalities and clinical predictors.

๑ 2021 Ahmad, Ahmed, Rajendraprasad, Loranger, Gupta, Velagapudi, Vivekanandan, Nahas, Plambeck, Douglas Moore. Published

by Elsevier Ltd on behalf of International Society for Infectious Diseases. 


\section{Transfer in die Praxis}

\section{Hintergrund}

Nach einer Infektion mit SARS-CoV-2 (severe acute respiratory syndrome coronavirus 2) kann in seltenen Fällen nach der akuten Infektionsphase ein multisystemisches inflammatorisches Syndrom (MIS) auftreten. Dies wurde zunächst bei Kindern und Heranwachsenden beschrieben (multisystem inflammatory syndrome in children, MIS-C) und kann einer Kawasaki-Erkrankung ähneln [1]. MIS-C tritt nicht vermehrt bei vorerkrankten Kindern auf. In 71\% der Fälle sind vier oder mehr Organsysteme betroffen, die häufig betroffenen Organsysteme sind gastrointestinal (92\%), kardiovaskulär (80\%), hämatologisch (76\%), mukokutan (74\%) und respiratorisch (70\%). Ein MIS-C kann schwer verlaufen, in einer Analyse von 186 Fällen sind 2\% der Kinder gestorben. 40\% der jungen Patienten wiesen Charakteristika einer Kawasaki-Erkrankung auf, bei 8\% wurden Aneurysmen der Koronararterien beschrieben [1]. Mit weiterer klinischer Erfahrung mit COVID-19 hat sich gezeigt, dass nicht nur bei Kindern, sondern auch bei Erwachsenen ein MIS auftreten kann (multisystem inflammatory syndrome in adults, MIS-A).

\section{Vorstellung des klinischen Falles}

Faran Ahmad und Kollegen präsentieren einen interessanten klinischen Fall zu einem erwachsenen Patienten mit MIS-A. Ein 26-jähriger Patient, der sich als Kontaktperson einer SARS-CoV-2-positiven Person in Quarantäne befunden hatte, stellte sich mit Fieber und Bauchschmerzen vor. In den Laboruntersuchungen waren deutlich erhöhte Entzündungswerte nachweisbar. Die Computertomographie (CT) des Thorax hatte nur leichte peribronchiale Verdickungen gezeigt, in der CT des Abdomens stellten sich vergrößerte Lymphknoten und ein Ödem in der Umgebung der Nieren dar. Im weiteren Verlauf kam es zu einer raschen klinischen Verschlechterung mit einem kardiogenen Schock bei schwergradiger biventrikulärer Funktionseinschränkung. Der Patient wurde komplex intensivmedizinisch behandelt. Hierzu zählte die Intubation und mechanische Beatmung, Vasopressoren, Implantation einer minimalinvasiven Herzpumpe (Impella $\left.{ }^{\circledR}\right)$ und Nierenersatzverfahren. Antiinflammatorisch erhielt der Patient intravenöse Immunglobuline, hochdosiertes Methylprednisolon, den Interleukin-1-Rezeptorantagonisten Anakinra sowie ASS. Nach 10 Tagen intensivmedizinischer Therapie konnte eine Erholung des Patienten erreicht werden.

\section{Fazit für die Praxis}

Nach den Kriterien des US-amerikanischen CDC (Center of Disease Control and Prevention; https://www.cdc.gov/mis/mis-a/hcp.html) kann die Diagnose MIS-A bei erwachsenen Patienten $\geq 21$ Jahre gestellt werden, die bestimmte klinische und laborchemische Kriterien erfüllen und für die eine andere Erkrankung als Erklärung unwahrscheinlich ist. Zu den klinischen Kriterien gehört neben Fieber eine schwere Herzerkrankung, Hautausschlag und Konjunktivitis, neurologische Symptome, Schock, Bauchschmerzen, Erbrechen oder Durchfall sowie eine Thrombozytopenie. Zu den laborchemischen Kriterien zählen erhöhte Entzündungswerte und der Nachweis einer aktuellen oder kürzlichen SARS-CoV-2-Infektion. In der thorakalen Bildgebung müssen nicht die für COVID-19-Pneumonie typischen Milchglastrübungen nachweisbar sein, häufiger sind peribronchiale Verdickungen und Pleuraergüsse. Bei MIS-A stehen die extrapulmonalen Manifestationen im Vordergrund. Bei der kardialen Beteiligung lässt sich in der kardialen Magnetresonanztomographie eine Myokarditis nachweisen [2]. Die Inzidenz von MIS-A ist ebenso wie die Pathogenese bisher unklar. MIS-A kann auch auftreten, wenn die SARS-CoV-2-Infektion inapparent verlaufen ist, wie in dem geschilderten Fall. Der mediane Zeitraum zwischen der SARS-CoV-2-Symptomatik und MIS-A beträgt 28 Tage [3]. Therapeutisch ist bei der Hälfte der Patienten eine intensivmedizinische Behandlung erforderlich. Für die Behandlung stehen entsprechend der Empfehlungen für MIS-C sowie nach vorliegenden Fallberichten vor allem intravenöse Immunglobuline, hochdosierte Steroide (Methylprednisolon) und bei therapierefraktären Verläufen Anakinra zur Verfügung. Außerdem kann in Analogie zu der Kawasaki-Erkrankung mit dem Risiko von Koronaraneurysmen ASS erwogen werden $[3,4]$. Unter Einsatz der Therapiemaßnahmen scheint eine rasche Verbesserung möglich zu sein, die mediane Krankenhausverweildauer wird mit 8 Tagen angegeben [3]. Es bleibt abzuwarten, ob auch die SARS-CoV-2-Impfung, wie in einem Fallbericht beschrieben, zu einem MIS führen kann [5]. Zusammenfassend muss ich an ein MIS-A denken, wenn nach einer COVID-19-Infektion ein inflammatorisches Syndrom mit extrapulmonalen Manifestationen, v.a. einer Herzerkrankung, auftritt.

\section{Disclosure Statement}

Es bestehen keine Interessenskonflikte.

\section{Literatur}

1 Feldstein LR, Rose EB, Horwitz SM, et al.: Multisystem Inflammatory Syndrome in U.S. Children and Adolescents. N Engl J Med. 2020;383:334-346.

2 Hékimian G, Kerneis M, Zeitouni M, et al.: Coronavirus Disease 2019 Acute Myocarditis and Multisystem Inflammatory Syndrome in Adult Intensive and Cardiac Care Units. Chest. 2021;159:657-662.

3 Patel P, DeCuir J, Abrams J, et al.: Clinical Characteristics of Multisystem Inflammatory Syndrome in Adults: A Systematic Review. JAMA Netw Open. 2021;4:e2126456.

4 Henderson LA, Canna SW, Friedman KG, et al.: American College of Rheumatology Clinical Guidance for Multisystem Inflammatory Syndrome in Children Associated With SARS-CoV-2 and Hyperinflammation in Pediatric COVID-19:Version 2. Arthritis Rheumatol. 2021;73:e13-e29.

5 Nune A, lyengar KP, Goddard C, et al.: Multisystem inflammatory syndrome in an adult following the SARS-CoV-2 vaccine (MIS-V). BMJ Case Rep. $2021 ; 14$.

Korrespondenz an:

Dr. Stefanie Keymel, stefanie.keymel@med.uni-duesseldorf.de 